\title{
HIV-1-negative female sex workers sustain high cervical IFNe, low immune activation, and low expression of HIV-1-required host genes
}

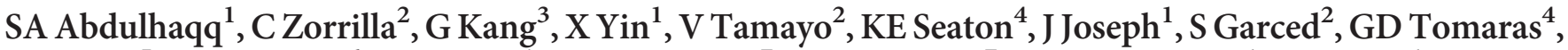 \\ KA Linn ${ }^{5}$, AS Foulkes ${ }^{6}$, L Azzoni ${ }^{1}$, M VerMilyea ${ }^{7}$, C Coutifaris $^{7}$, AV Kossenkov ${ }^{1}$, L Showe ${ }^{1}$, EN \\ Kraiselburd $^{8}, \mathrm{Q} \mathrm{Li}^{3}$ and LJ Montaner ${ }^{1}$
}

Sex workers practicing in high HIV endemic areas have been extensively targeted to test anti-HIV prophylactic strategies. We hypothesize that in women with high levels of genital exposure to semen changes in cervico-vaginal mucosal and/or systemic immune activation will contribute to a decreased susceptibility to HIV-1 infection. To address this question, we assessed sexual activity and immune activation status (in peripheral blood), as well as cellular infiltrates and gene expression in ectocervical mucosa biopsies in female sex workers (FSWs; $n=50$ ), as compared with control women (CG; $n=32$ ). FSWs had low-to-absent HIV-1-specific immune responses with significantly lower CD38 expression on circulating $\mathrm{CD}^{+}{ }^{+}$or $\mathrm{CD}^{+}$T-cells (both: $P<0.001$ ) together with lower cervical gene expression of genes associated with leukocyte homing and chemotaxis. FSWs also had increased levels of interferon- $\varepsilon$ (IFNع) gene and protein expression in the cervical epithelium together with reduced expression of genes associated with HIV-1 integration and replication. A correlative relationship between semen exposure and elevated type-1 IFN expression in FSWs was also established. Overall, our data suggest that long-term condomless sex work can result in multiple changes within the cervico-vaginal compartment that would contribute to sustaining a lower susceptibility for HIV-1 infection in the absence of HIV-specific responses.

\section{INTRODUCTION}

Women comprise $>50 \%$ of HIV-1-infected individuals, with heterosexual transmission representing the leading route for infection. ${ }^{1}$ Understanding the factors that contribute to HIV-1 transmission in the cervico-vaginal compartment is essential for the development and improvement of HIV vaccines and/or preexposure prophylaxis approaches. Several factors have been associated with higher rates of HIV infection in women. These include preexisting inflammation, as well as repeated exposure via commercial sex work. ${ }^{2,3}$ However, studies in high HIV prevalence areas have also identified female sex workers (FSWs) who remain seronegative despite long-term sexual work and low condom use (highly exposed seronegative, HESN). These observations led to the hypothesis that these women may have developed intrinsic or adaptive mechanism(s) of resistance. ${ }^{4}$ Cell-mediated adaptive responses in peripheral blood ${ }^{4}$ and vaginal mucosal anti-HIV immunoglobulin A $(\operatorname{IgA})^{5}$ titers are frequently absent or weak in HESN FSWs, and in one study did not exclude subsequent seroconversion. ${ }^{6}$ Thus, adaptive anti-HIV-1-specific responses are unlikely to be solely responsible for maintaining an HESN status.

Recent studies, largely supported by data in peripheral blood or cervico-vaginal secretions, suggest that HESN FSWs may sustain a state of immune quiescence or reduced immune

${ }^{1}$ The Wistar Institute, Philadelphia, Pennsylvania, USA. ${ }^{2}$ Maternal-Infant Study Center (CEMI), University of Puerto Rico, San Juan, Puerto Rico. ${ }^{3}$ School of Biological Sciences and Nebraska Center for Virology, University of Nebraska, Lincoln, Nebraska, USA. ${ }^{4}$ Duke Human Vaccine Institute, Duke University, Durham, North Carolina, USA. ${ }^{5}$ Department of Biostatistics and Epidemiology, University of Pennsylvania, Philadelphia, Pennsylvania, USA. ${ }^{6}$ Division of Biostatistics and Epidemiology, University of Massachusetts, Amherst, Massachusetts, USA. ${ }^{7}$ University of Pennsylvania Health System, Philadelphia, Pennsylvania, USA and ${ }^{8}$ Department of Microbiology, UPR Medical School, San Juan, Puerto Rico. Correspondence: LJ Montaner (montaner@wistar.org) 
activation that can counter infectivity in spite of repeated acute HIV-1/semen exposures. ${ }^{7-10}$ Importantly, it has remained unknown to what extent semen vs. other factors (e.g., repeated HIV-1 exposure) may contribute to these observed changes of the cervical tissue microenvironment and in systemic immune modulation. Additional mechanisms proposed to maintain HESN status include genetic polymorphism, ${ }^{11}$ increased peripheral T-regulatory cell frequency, ${ }^{7}$ and an increase in antiproteases within the cervico-vaginal mucosa. ${ }^{12}$

Low condom use consistently exposes HESN FSW s to semen and HIV-1. Human semen, more than an inert vehicle for HIV1 virions within the female reproductive tract, has been shown to be immunologically pleiotropic. Studies have shown that semen can acutely upregulate inflammatory cytokines and chemokines (e.g.: interleukin (IL)-6, IL-8, C-C chemokine ligand 20, and $\mathrm{C}-\mathrm{X}-\mathrm{C}$ chemokine ligand 3 ) in cervico-vaginal tissue-derived epithelial cell lines and induce infiltration of immune effectors into cervico-vaginal tissue shortly after coitus. ${ }^{13,14}$ However, semen also includes factors able to mediate a tolerogenic $\mathrm{T}$ helper type 2 (Th2) profile (e.g., T-regulatory cell induction) driven by high levels of IL-10, transforming growth factor- $\beta$, and prostaglandin E2 in semen, which have been hypothesized to have an important role during fertilization. ${ }^{15-18}$ Aside from effects of repeated signaling by IL10 or prostaglandin E2 on myeloid and T-cells in the cervix, the presence of Th2-polarized CD4s alone would not exclude infection as both Th1 and Th2 CD4 cells are susceptible to
HIV-1. ${ }^{19}$ Although seminal fluid-derived amyloid fibrils have been shown to have variable results in affecting $\mathrm{HIV}-1$ infectivity in vitro, ${ }^{20}$ no marked in vivo effects were noted when tested in non-human primates for acute effects on simian immunodeficiency virus (SIV) infection. ${ }^{21}$ Independently of semen, work on non-human primate models has shown that acute exposure to high-titer SIV can also induce recruitment of CD4 T-cells, plasmacytoid dendritic cells (DCs), and macrophages into the cervical and vaginal epithelium that if followed by infection can result in a greater depletion of CD4 T-cells. ${ }^{22,23}$ In spite of the potential acute effects of semen and/or viral particle exposure, analysis of ectocervical tissue from HESNs has shown a steady state of reduced rather than increased inflammation, ${ }^{24}$ suggesting that the tissue microenvironment alterations resulting from chronic semen or viral particle exposure (e.g.: from long-term sex work) may differ from those associated with acute semen/viral exposure.

In order to assess the contribution of sex work in groups with low HIV exposure incidence, we hypothesized that in women with high levels of genital exposure to semen, both localized tissue (i.e.: cervico-vaginal mucosa) and/or systemic immune activation changes will contribute to a lower susceptibility for HIV-1 infection. Furthermore, as no report outside Africa has yet described blood-based and cervical tissue changes in longterm sex worker cohorts, it also remains unknown whether reported HESN or long-term sex work protective mechanisms, such as immune quiescence, are attributable to differential

Table 1 Sexual practices and cervico-vaginal status among biopsy contributors

\begin{tabular}{|c|c|c|c|}
\hline Category & Control group (CG), 35 women & FSW, 50 women & $\boldsymbol{P}$-values \\
\hline Age (years) & $35.5(29.5-41.25)$ & $32(28-38)$ & 0.439 \\
\hline Unprotected sex (1 week) & $0(0-3)$ & $3(2-9.7)$ & $<0.0001^{\mathrm{a}}$ \\
\hline Years of sex work & $(\mathrm{NA})$ & $8.45(5-11.5)$ & \\
\hline Sterilization ${ }^{\mathrm{b}}$ & $4(17.1 \%)$ & $27(54 \%)$ & $0.0025^{\mathrm{a}}$ \\
\hline Non-IDU drug use & $4(12.9 \%)$ & 34 (68\%) & $<0.0001^{a}$ \\
\hline \multicolumn{4}{|l|}{ Vagina microenvironment } \\
\hline Chlamydia $^{\mathrm{c}}$, Gonorrhea, Syphilis & $0(0 \%)$ & $8(16.0 \%)$ & $0.0247^{a}$ \\
\hline
\end{tabular}

Hormone levels

Abbreviations: ASC, atypical squamous cell; CIN, cervical intraepithelial neoplasia; FSW, female sex worker; HPV, human papillomavirus; IDU, injection drug use. Parenthetical data are presented as range or as percentage.

andicates significant variable as measured by Mann-Whitney $U$ or Fisher's Exact Test.

bSterilization was done by tubal ligation.

'Sexually transmitted infections (STIS) were asymptomatic; symptomatic STIs were a part of the exclusion criteria.

dFor estradiol: 28 FSWs/14 CG measured. 
Table 2 Selected significant variables from flow cytometric analysis of PBMC

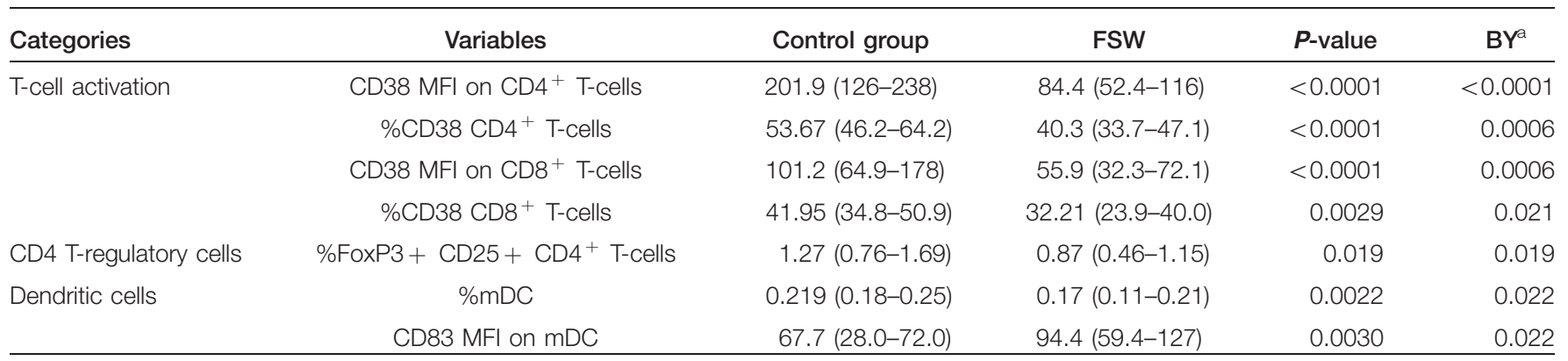

Abbreviations: BY, Benjamini and Yekutieli; FSW, female sex worker; mDC, myeloid dendritic cell; MFI, mean fluorescent intensity; PBMC, peripheral blood mononuclear cell. Variables are listed in order of $P$-value. Median and interquartile range values are shown. Group comparisons were carried out by Mann-Whitney $U$-tests.

${ }^{a}$ Benjamini and Yekutieli adjustment (at the rate of 10\%) was carried out per examined immune cell compartment or functional test.

genetics or regional sex worker practices. To address our stated hypothesis and reproducibility of mechanisms associated with long-term sex work, we analyzed a cohort of seronegative longterm FSWs (and a comparator group (CG) of women with low semen exposure, as determined by self-reported frequency of unprotected intercourse) recruited from a geographical distinct region, San Juan, Puerto Rico. HIV-1 prevalence among FSWs in San Juan has been previously reported at $4.8 \%,{ }^{25}$ which is 515 -fold lower than in sub-Saharan Africa. ${ }^{26}$ Although Puerto Rican FSWs cannot be directly compared with HESN FSWs African cohorts, if semen (and not HIV exposure itself) is responsible for the mucosal changes observed in HESN FSWs in Africa, we would expect that FSWs with no HIV-specific immune responses but high levels of condomless sex (semen exposure) will have comparable peripheral and mucosal changes as described in HESN FSWs.

\section{RESULTS}

\section{Study population}

The cohort characteristics are reported in Table 1. The median ages of the FSWs $(n=50)$ and CG $(n=35)$ were 35.50 and 32.00 years, respectively $(P=0.439$; Table 1$)$. In all, $44(88 \%)$ FSWs reported a sex work duration of $\geqslant 3$ years. CG women were sexually active women in stable monogamous relationships attending a women's health clinic at the study site. In the week prior to sample collection, FSWs reported a median of three events of condomless vaginal intercourse, compared with zero among CG women $(P<0.0001)$. Only 2 (4\%) of FSWs reported use of oral hormonal-based contraceptives, as compared with $15(43 \%)$ of the CG $(P<0.0001)$. Only one woman within the control reported use of injectable contraceptives. Despite this difference in contraceptive use, serum levels of estradiol and progesterone were not significantly different between the two groups. Although intravenous drug users were excluded from recruitment, 27 (54\%) FSWs reported other drug use (marijuana). Although symptomatic sexually transmitted infections (STI) were disqualifiers for the study, enrollment screening identified 8 (16\%) FSWs with asymptomatic STIs as summarized in Table 1. Thirty-four (75.6\%) FSWs vs. 21
(61.7\%) of the CG had a vaginal $\mathrm{pH}$ of $\geqslant 4.5$. There were no significant differences in human papillomavirus infection or abnormal cytology rates between the two groups.

\section{Rare HIV-1-specific responses in FSWs with low T-cell CD38 expression}

Gag-specific T-cell responses and cervico-vaginal HIV-1specific IgA/IgG antibody levels were measured as indicators of HIV-1 exposure in the absence of infection. ${ }^{5,27}$ As expected, owing to the low HIV prevalence in area, anti-HIV-1 cellular responses as assessed by intracellular staining for interferon- $\gamma$ (IFN $\gamma)$ and extracellular staining for CD107a after peptide stimulation to a consensus gag sequence were extremely low (2 of the 47 FSWs; see Supplementary Figure S1a online). With regard to humoral anti-HIV responses, only 2 FSWs had detectable mucosal HIV-1-specific IgA or IgG, as assessed by having anti-HIVspecific activity for $>2$ HIV-1-specific antigens in the binding antibody multiplex assay (see Supplementary Figure S1b). Taken together, $<10 \%$ of FSWs but no control had a HIV-1-specific response.

In contrast to HIV-specific responses, flow cytometry-based analysis of activation and functional responses in circulating T-cell, natural killer, and DCs evidenced a lower expression of CD38 in both $\mathrm{CD}^{+}(P<0.00001)$ and $\mathrm{CD} 8{ }^{+}$T-cell subsets $(P=0.0006)$ (Table 2 ; see Supplementary Table $\mathbf{S 1})$, indicating that the T-cell compartment was modulated in the FSW cohort. Importantly, the difference in CD38 expression remained significant after adjustment for confounding factors (drug use, oral contraception, sterilization, marijuana use; see Supplementary Table S2), supporting its association with long-term sex work. A lower frequency of regulatory $\mathrm{CD} 4^{+}$ $\mathrm{T}$-cells and an increased expression of maturation marker CD83 on myeloid DCs were also detected in FSWs (Table 2). We did not observe any significant between-groups difference in natural killer cell (lysis of K562 cells) or myeloid (ex vivo Toll-like receptor 3-, 4-, or 9-mediated cytokine responses by peripheral blood mononuclear cells (PBMC)) functional responses (not shown). Taken together, FSWs showed changes in T-cell subsets indicative of peripheral T-cell modulation of CD38 expression as described in HESN cohorts. ${ }^{7,28,29}$ 

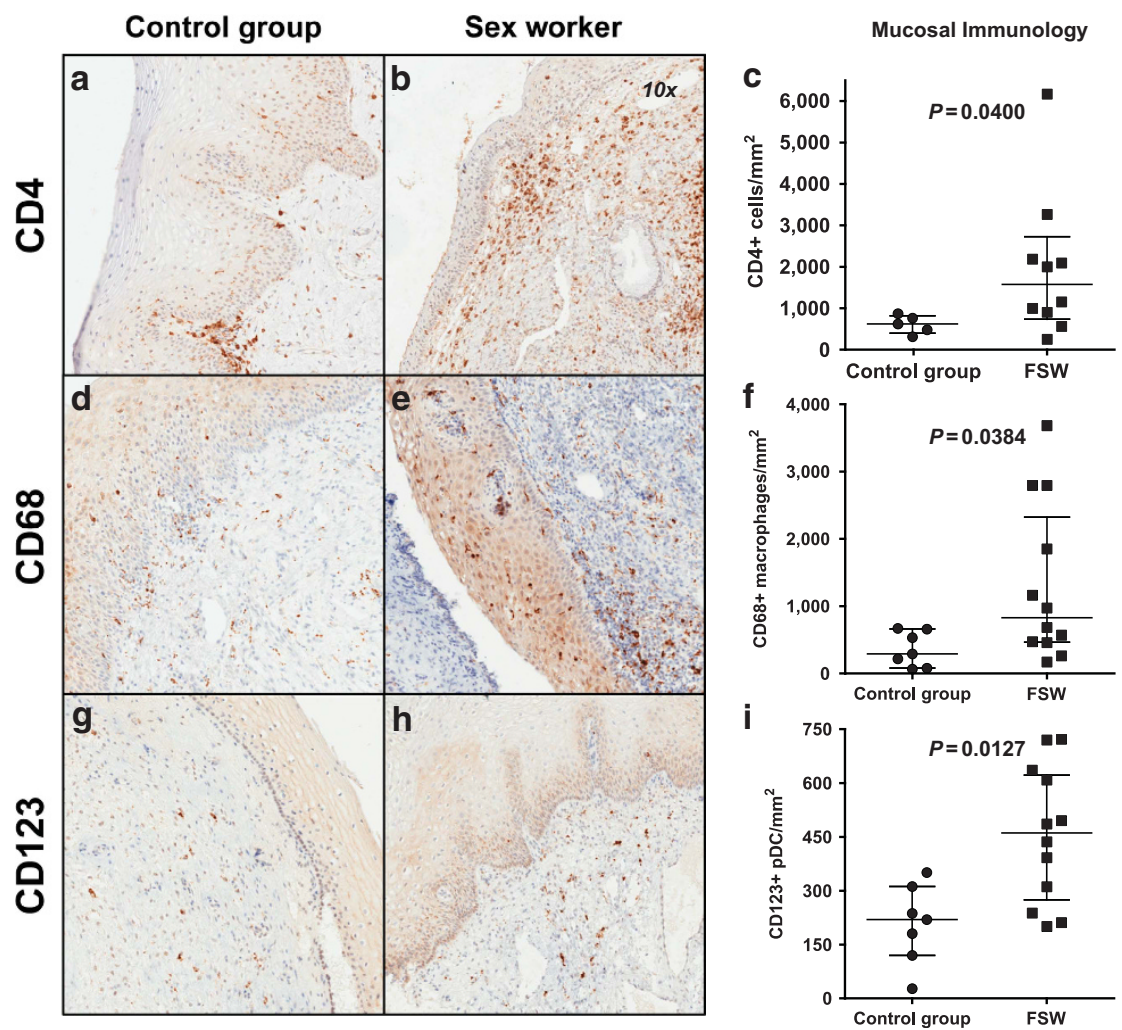

Figure 1 Increased levels of immune effectors in female sex worker (FSW) ectocervical tissue as compared with control women. Pictured are immunohistochemical images from each staining group representative of control or sex worker groups; brown punctate staining represents individually stained cells. (a-c) FSWs had over $2 \times$ more CD4 ${ }^{+} \mathrm{T}$-cells within ectocervical biopsies as compared with biopsies from low-risk controls (median: FSWs 1958 vs. control grouo (CG) 609.3 cells mm ${ }^{-2} ; P=0.0400$ ). (d-f) $C D 68^{+}$macrophages were also overrepresented in FSWs with the highest differential between FSWs and CG women (median: FSWs 829.6 vs. CG 294.0 cells $\mathrm{mm}^{-2} ; P=0.0384$ ). (g-i) FSWs had higher levels of CD123 ${ }^{+}$plasmacytoid dendritic cells ( $\mathrm{pDC}$ ) within ectocervical tissue as compared with controls (median: FSWs 461.1 vs. CG 219.8 cells $\mathrm{mm}^{-2} ; P=0.0127$ ). Images at $\times 10$. Median and interquartile range are shown in c, $\mathbf{f}$, and i. Groups comparison was carried out by Mann-Whitney U-tests.

\section{Higher immune cell infiltrates in ectocervical mucosa biopsies from FSWs}

We next examined CD4 ${ }^{+}$T cells, CD $123^{+}$plasmacytoid DCs and $\mathrm{CD} 8^{+}$macrophage infiltrates in 29 ectocervical biopsies (targeted to the squamo-columnar junction region of the cervix) from a subset of FSWs $(n=16)$ and CG $(n=13)$. Subject variables for this subset were comparable to the full cohort (see Supplementary Table S3), with the exception that no difference in STI frequency was detected.

$\mathrm{CD} 4{ }^{+}$cells (Figure 1a-c) were over $2 \times$ more abundant in FSW biopsies as compared with controls (median: FSWs 1958 vs. CG 609.3 cells $\left.\mathrm{mm}^{-2} ; P=0.0400\right)$. Interestingly, the CD4 ${ }^{+}$ $\mathrm{T}$-cell infiltrate was predominantly located in the lamina propria, with many cells adjacent to the basal membrane.

CD68-positive cells, consistent with macrophage infiltrates (Figure 1d-f), were also increased in FSWs (median: FSWs 829.6 vs. CG 294.0 cells $\mathrm{mm}^{-2} ; P=0.0384$ ) and were broadly distributed in both the lamina propria and within the epithelial layer.

Finally, FSWs had significantly higher amounts of CD123 ${ }^{+}$ cells (plasmacytoid DCs, Figure 1g-i) within the lamina propria

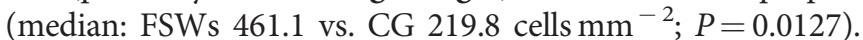
Taken together, our data indicate that FSWs had significantly increased levels of cervico-vaginal mucosa immune CD4 T-cell and myeloid infiltration.

\section{Gene expression in ectocervical biopsies}

To assess the tissue microenvironment associated with the observed cell infiltrates, we measured global gene expression in ectocervical biopsies from a subset of 26 women ( 11 control and 14 FSWs) using HumanHT-12 v4 BeadChips microarrays. Of the 29,377 probes expressed in the samples, 873 probes were found to be significantly different between control and FSW groups (false-discovery rate $<20 \%$ ). Over $70 \%$ of the differentially expressed genes were upregulated in FSWs. Principal Component Analysis confirmed that differential gene expression from FSWs and CG clustered into two distinct sample groups (Figure 2a). Ingenuity Pathway Analysis of the 873 differentially expressed genes did not reveal an enrichment of immune activation gene networks within the FSW cohort and instead indicated the presence of a functional inhibition of leukocyte homing $(P=0.00036)$ or chemotaxis $(P=0.00029)$ (Table 3 ), suggesting resident regulation against added cell recruitment.

Genes associated with resistance to viral infection (33 genes, Figure 2b) or susceptibility to viral infection (60 genes, Figure 2c) were differential between FSWs and CG with analysis, indicating gene modulation within FSWs favoring viral susceptibility (Figure 2). To further target our analysis to IFN-mediated signatures, we compared our significant gene 

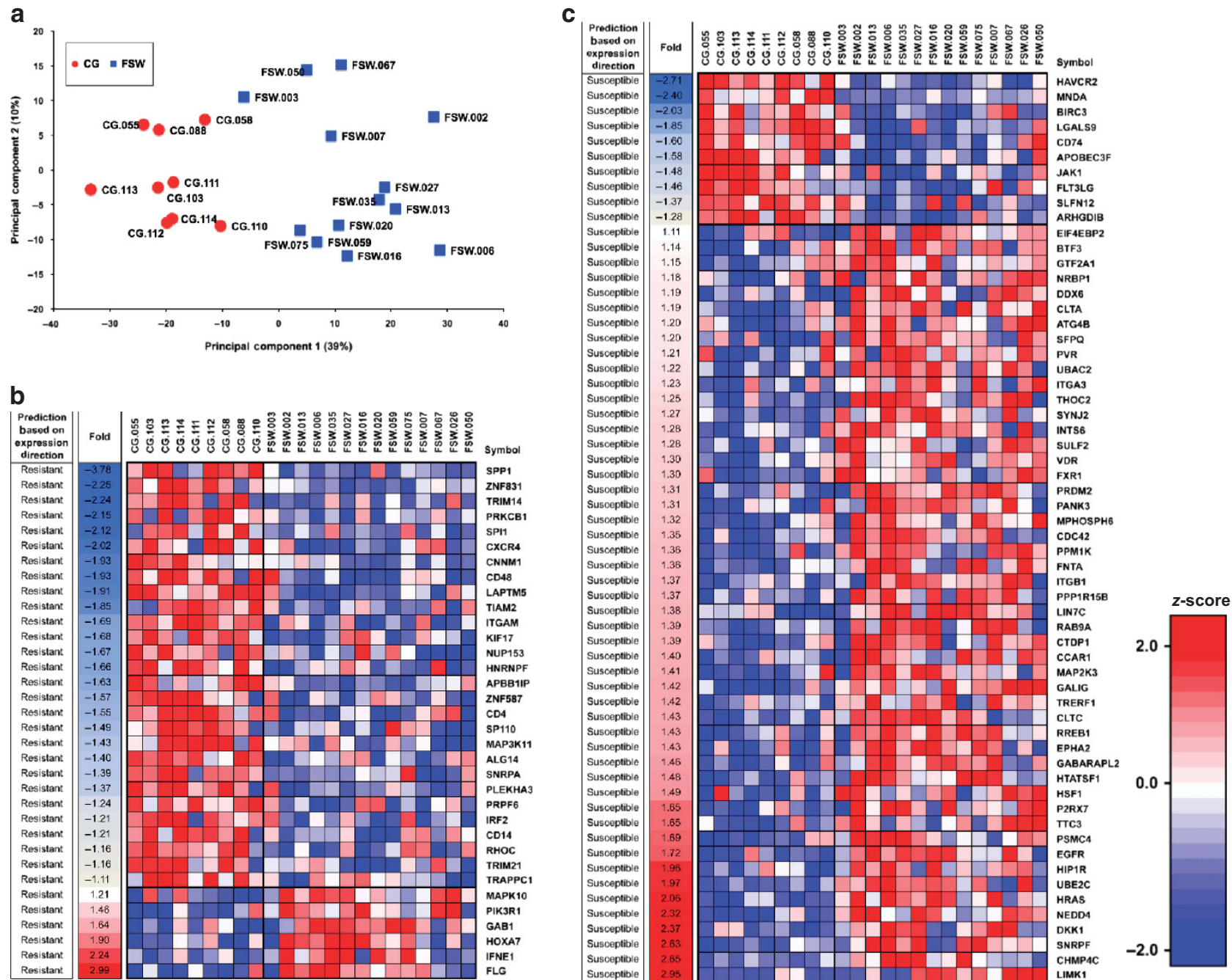

Figure 2 Gene expression differences in ectocervical biopsies between 11 females from control group (CG) and 14 female sex workers (FSWs). (a) Principle component analysis using all 873 significant genes $(1.2 \geqslant$ fold) demonstrates clear segregation of sex workers from CG samples. (b) Of the 873 genes, 106 overlapped with a predetermined Ingenuity Pathway Analysis (IPA) list of genes involved with viral infection. Thirty-three of the 106 genes differentially regulated between FSWs and CG had an expression profile in FSWs predicted to reduce susceptibility to viral infection as compared with CG. (c) Conversely, 60 of the 106 genes had an expression profile predicted to increase susceptibility to viral infection in FSWs as compared with CG. Overall, IPA predicted higher viral susceptibility in FSWs as compared with CG $(Z$-score $=+3.35)$.

expression data to the Interferome database. ${ }^{30}$ Of the 873 significantly modulated genes, $95(10 \%)$ were identified as IFN-regulated genes. Of these, 39\% (37 genes) were associated with a type I IFN stimulation (see Supplementary Figure S2), documenting the presence of a type I IFN signature, yet without evidence for a highly polarized response. A similar result was observed upon examination of the 102 type II IFN-regulated genes in the gene data set. Of these, only $35 \%$ were associated with an increased IFN $\gamma$ response (data not shown).

Taken together, our tissue-based RNA microarray data indicate that FSWs do not have a localized enhanced immune activation signature, despite the observed greater mucosal immune cell infiltrates; this is consistent with an immune quiescent cervico-vaginal microenvironment.

\section{IFN $\varepsilon$ staining of ectocervical biopsies}

Among viral susceptibility genes (Figures $\mathbf{2} \mathbf{b}$ and $\mathbf{3 b}$ ), we noted that IFN $\varepsilon$ was significantly overexpressed (2.2 times) in biopsies from FSWs, as compared with the CG. IFNE is a novel type I IFN preferentially produced by epithelial cells of the female reproductive tract and implicated in protection from both viral and bacterial STIs. ${ }^{31,32}$ Immunohistochemical staining confirmed the elevated IFN $\varepsilon$ protein expression in FSWs; this was restricted to the basal and parabasal layers of stratified squamous epithelium of the ectocervix (Figure 3a). In biopsies that captured columnar cells of the squamo-columnar junction, IFN $\varepsilon$ was also present within the columnar cells (data not shown). Quantification of IFN $\varepsilon$ protein expression within the epithelial layer indicated that FSWs had protein levels $2 \times$ higher than controls $(P=0.0336$; Figure $3 \mathrm{c})$. IFN $\varepsilon$ protein 
Table 3 Biological functions significantly altered based on genes differentially expressed between FSW and control group women

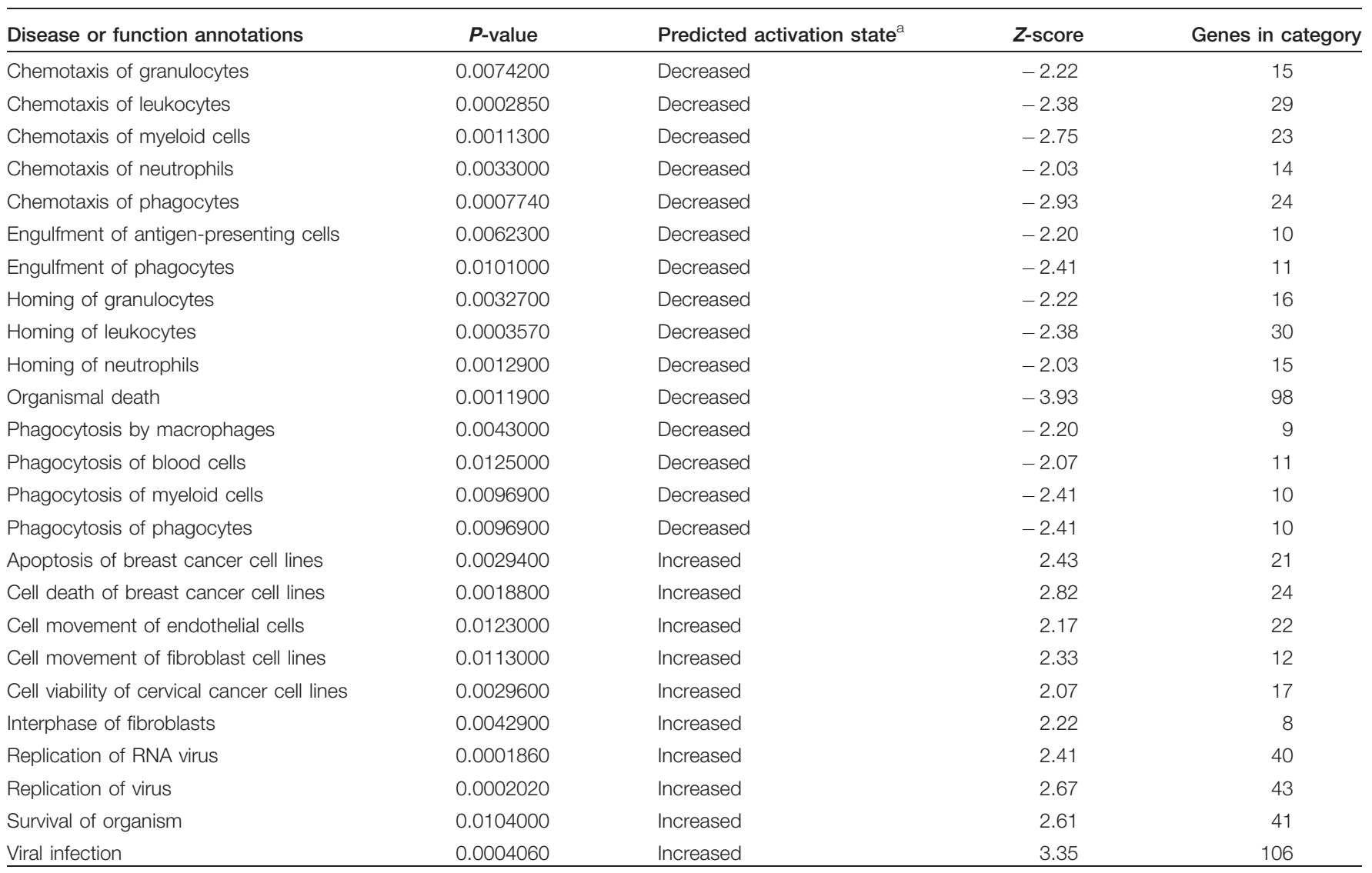

Abbreviation: FSW, female sex worker.

${ }^{a}$ Activation state indicates direction of change in FSWs compared with control group, with the significance of the prediction expressed as Z-score.

levels were positively correlated with levels of gene expression $(r=0.6954, P=0.0019$; Figure 3d).

In contrast to other type I IFNs induced by pattern recognition receptors, IFNE expression has been reported to be regulated by estrogen levels. ${ }^{31}$ However, we were unable to detect any significant difference in plasma estrogen levels between our study groups, and we found no significant correlation between estrogen levels and IFN $\varepsilon$ gene expression $(r=0.352, P=0.108)$ or protein levels $(r=0.305, P=0.19)$. IFN $\varepsilon$ gene expression has also been shown to be regulated by semen exposure in murine models ${ }^{31}$ and human ectocervical cell lines. ${ }^{14}$ We therefore reanalyzed data by stratifying our study participants based on self-reported condom use in the week prior to sampling (irrespective of prior FSW or CG assignment). The reanalysis resulted in detection of a greater difference with women who reported unprotected intercourse in the prior week showing higher $(\sim 3 \times)$ IFN $\varepsilon$ expression than women reporting abstinence or consistent condom use $(P=0.001$; Figure 3e) and showed that self-reported condomless sexual activity correlated with IFN $\varepsilon$ expression $(r=0.4907, P=0.024)$. Of interest to acute effects of semen, we did detect higher levels of IL-1 $\beta$, IL-6, and IL-8 in cervico-vaginal secretions from women grouped by condomless sex criteria (see Supplementary Figure S3); of note, these differences were not observed comparing FSW vs. CG as there were no differences detected among all variables tested (data not shown). Reanalysis of all previously described significant variables by this new grouping did not result in detection of differences across all variables except IFN $\varepsilon$ tissue expression consistent with their association with longterm sex work rather than short-term semen exposure (see Supplementary Table S2). Similarly, a reanalysis of gene expression data based on condomless sex did not detect a single gene as significant below the $20 \%$ false-discovery rate cutoff. To allow for the inference as to whether or not semen exposure could increase IFN $\varepsilon$ RNA and protein expression in humanderived cervical epithelium, we tested the effects of in vitro semen exposure on the ectocervical cell line ECT1. A 6-h incubation with $5 \%$ seminal plasma resulted in a 2.5 -fold increase in IFNe gene expression $(P=0.0023$; see Supplementary Figure S4a) in conjunction with higher protein expression (see Supplementary Figure S4b). Taken together, semen-inducible IFN $\varepsilon$ expression is present at a higher level within the epithelial layer of the cervix in women reporting condomless vaginal intercourse. 

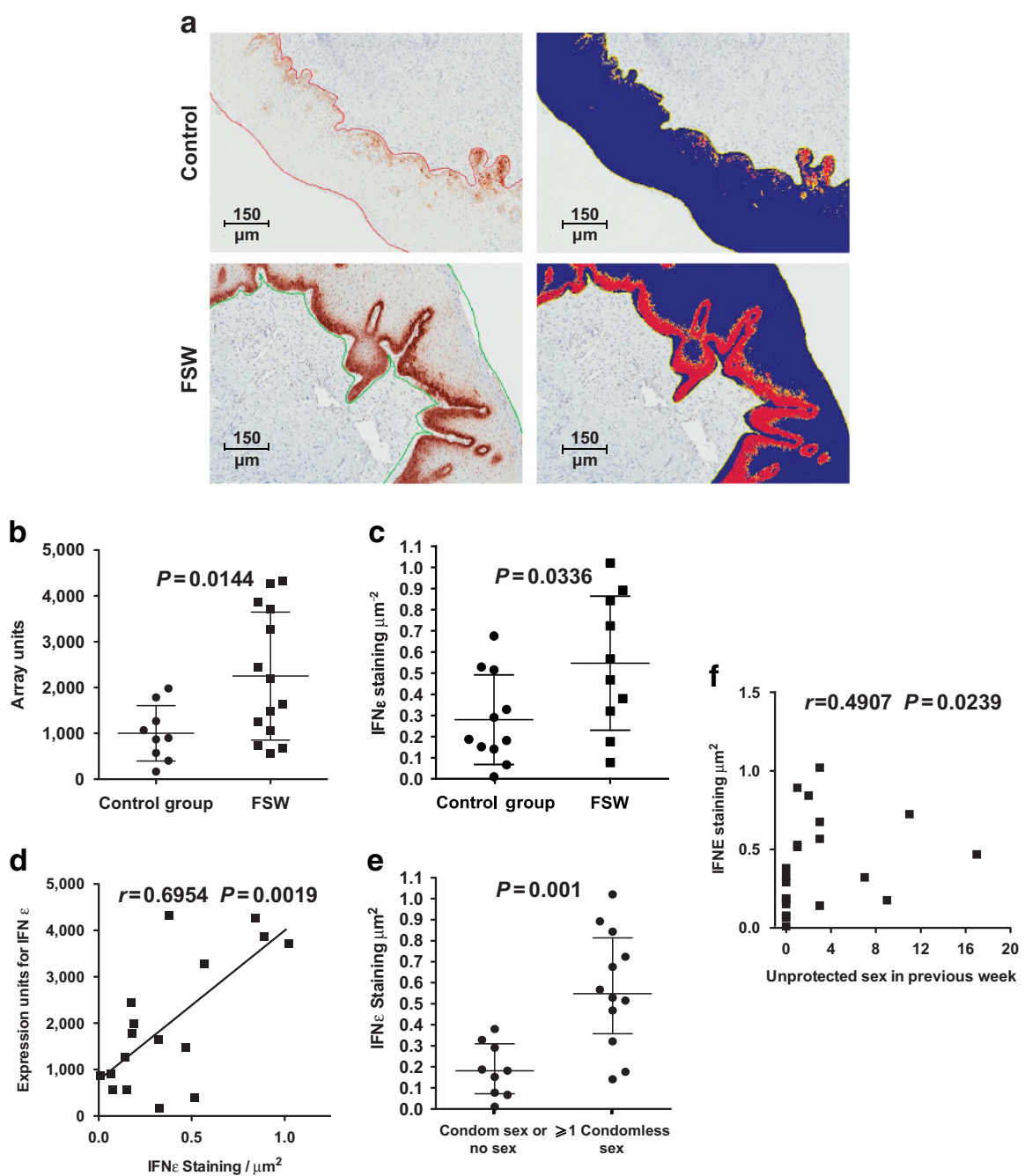

Figure 3 Increased interferon- $\varepsilon$ (IFN $\varepsilon$ ) in female sex worker (FSW)-derived ectocervical tissue as compared with samples from women in the control (a) IFN $\varepsilon$ staining (brown) showed that expression of this type I IFN was tightly restricted to basal and parabasal stratified squamous epithelium. Staining was also evident in columnar cells but to a much lesser extent (data not shown). FSWs (bottom) had much higher expression of IFN $\varepsilon$ than controls (Top) in general. (b) Both gene expression array analysis (right panels are gradient maps to highlight positive staining within the epithelium) $(P=0.0144)$ and $(\mathbf{c})$ image quantification $(P=0.0336)$ of tissues showed higher expression of IFN $\varepsilon$ in FSWs. (d) Protein expression for IFN $\varepsilon$ strongly correlated with the gene expression array $(r=0.6954 P=0.0019)$, showing that FSWs had higher IFN $\varepsilon$ expression. (e) Splitting all women in the study based on previous unprotected sex in the week prior to biopsy showed that women who had unprotected sex had $3 \times$ more IFN $\varepsilon$ than those who did not $(P=0.001)$. (f) IFN $\varepsilon$ in cervical tissue correlated with frequency of condomless sex in the week prior to sampling $(r=0.4907 P=0.0239)$. Median and interquartile range are shown in $\mathbf{a}, \mathbf{b}$, and $\mathbf{e}$. The difference between groups was tested using Mann-Whitney tests. Correlations were assessed using Spearman test.

\section{Downregulation of genes required for HIV-1 infection in sex workers}

As the host profile of gene expression required for HIV-1 infection and replication has been well studied, we assessed whether the ectocervical tissue of FSWs would express differential levels of target genes as previously defined by various functional genomic screens or genome-wide association studies (Supplementary Table S4). ${ }^{33-36}$ Our analysis identified 23 genes, differentially expressed in FSWs as compared with controls that overlapped with results from these prior studies (Table 4 and Figure 4). The degree of overlap was significantly higher than by random chance alone, as determined by hypergeometrical testing for three of the four studies examined (see Supplementary Table S4); moreover, we found that the degree of overlap with genes identified in two or more of the screens was highly significant $(P<0.0001$; see Supplementary Table S4). These downregulated genes included CD4 and Nucleoporin 153, which have been shown to be critical for nuclear import of HIV and integration. ${ }^{37}$ Overall, target gene expression indicates that lower levels of genes required for HIV infection are present in ectocervical tissue from FSWs.

\section{DISCUSSION}

We show that in long-term HIV seronegative FSWs, three distinct, non-adaptive mechanisms may contribute to decreasing susceptibility to HIV-1 infection: lower mucosal tissue immune activation, enhanced epithelial IFN $\varepsilon$ expression, and lower expression of HIV-permissive host genes. With regard to enhanced epithelial IFN $\varepsilon$ expression, we further show that 
Table 4 Genes from sex worker ectocervical biopsy microarray that overlap with genes required for HIV infection

\begin{tabular}{|c|c|c|c|}
\hline Gene names & Fold & $P$-value & FDR \\
\hline C-X-C chemokine receptor type 4 (CXCR4) & -2.02 & 0.00370 & $17.6 \%$ \\
\hline T-cell lymphoma invasion and metastasis 2 (TIAM2) & -1.85 & 0.00406 & $17.6 \%$ \\
\hline Nucleoporin 153 kDa (NUP153) & -1.67 & 0.00251 & $15.5 \%$ \\
\hline Cluster of differentiation 4 (CD4) & -1.55 & 0.00054 & $8.2 \%$ \\
\hline SP110 nuclear body protein (SP110) & -1.49 & 0.00220 & $15.5 \%$ \\
\hline Janus kinase 1 (JAK1) & -1.48 & 0.00127 & $10.8 \%$ \\
\hline Four-phosphate-adaptor protein 1 (FAPP1) & -1.37 & 0.00111 & $10.8 \%$ \\
\hline Trafficking protein particle complex (TRAPPC1) & -1.11 & 0.00407 & $17.6 \%$ \\
\hline Carboxy-terminal domain, RNA polymerase II, polypeptide A, phosphatase, subunit 1 (CTDP1) & 1.39 & 0.00816 & $15.5 \%$ \\
\hline Gamma-aminobutyric acid receptor-associated protein-like 2 (GABARAPL2) & 1.46 & 0.00222 & $10.8 \%$ \\
\hline HIV-1 Tat-specific factor 1 (HTATSF1) & 1.48 & 0.00272 & $10.8 \%$ \\
\hline Epidermal growth factor receptor (EGFR) & 1.72 & 0.00099 & $8.2 \%$ \\
\hline Huntingtin-interacting protein 1-related protein (HIP1R) & 1.96 & 0.01007 & $15.5 \%$ \\
\hline
\end{tabular}

Abbreviation: FDR, false-discovery rate.
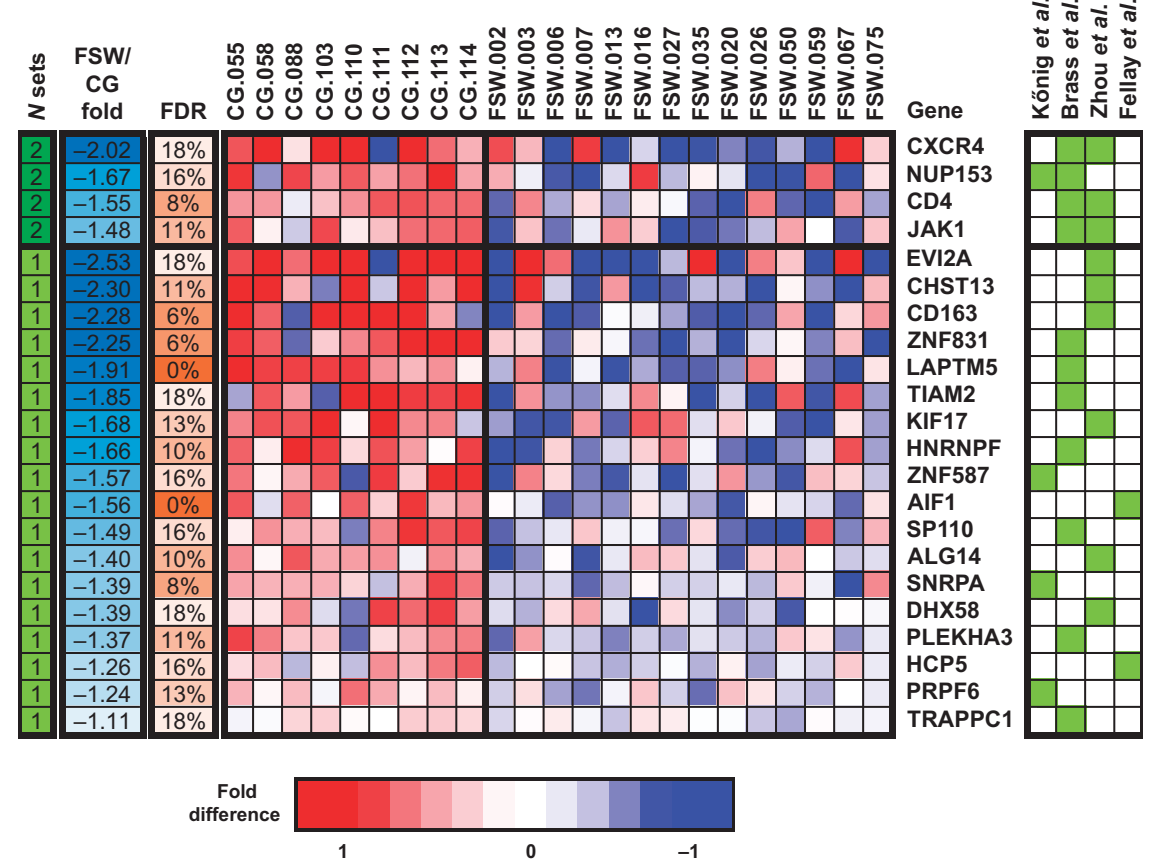

Figure 4 Heatmap of dowregulated genes from female sex worker (FSW)/control group (CG) microarray shown previously to be essential for HIV-1 infection and replication. As multiple studies have examined required genes for HIV-1 infection/replication, we assessed our data set for overlap with four previously published gene sets. ${ }^{33-36}$ Twenty-two genes from our data set overlapped with results from these prior studies (hypergeometrical test: $P<0.0001$ ); this was more than twice the number of genes as expected from random overlap. Additionally, being present in at least two of these prior studies further validated four of these genes. Heatmap is presented as fold difference over median expression across all patients for a particular gene. HIV sets are color coded as green for overlap with a particular data set and white for no overlap. 
semen exposure can contribute to the induction of IFN $\varepsilon$ in cervical cells in vitro, connecting for the first time a feature of long-term sex work (repeated semen exposure through condomless intercourse) with the induction of a highly localized type I IFN in the cervico-vaginal epithelium. Our results indicate that IFN $\varepsilon$ mRNA and protein are co-expressed in the human female reproductive tract and are upregulated in women with high exposure to semen, suggesting semen components may regulate gene and protein expression of IFN $\varepsilon$ (otherwise regulated by estrogen levels in females ${ }^{31}$ ) in this compartment, inclusive of transcriptional induction. Our analysis identifies a previously uncharacterized immunological impact of condomless sexual activity of relevance to high-risk groups where HIV-1 prophylactic interventions are usually first tested.

Our observations on CD38 expression on T-cells within PBMC of long-term FSWs provide similar results as previous reports (based on African and European cohorts $^{28,29}$ ) of lowered T-cell activation (i.e.: CD38 expression) in HESN FSWs. Importantly, we now document that this change in circulating T-cell is not unique to HESN FSW cohorts, but associated with long-term sex work. The mechanism mediating a reduced peripheral blood T-cell CD38 expression in FSWs remains to be elucidated. In HESNs, CD38 expression on $\mathrm{CD} 4{ }^{+} \mathrm{T}$-cells has been reported to be negatively correlated with condom use. ${ }^{28}$ Thus it is tempting to speculate that semen may impact systemic T-cell activation. The concept that semen can have systemic effects is supported by other observations that semen exposure can drive gene regulation and systemic changes outside of the female reproductive tract. ${ }^{38}$ However, in our cohort, analysis performed based on recent semen exposure (i.e.: recent condomless intercourse) did not efficiently segregate $\mathrm{CD} 38$ expression on circulating $\mathrm{CD} 4^{+}$or $\mathrm{CD} 8{ }^{+}$ T-cells. We hypothesize this systemic change may be due to long-term rather than short-term sex work (or long term semen exposure). Indeed, after adjusting for confounders present at baseline, differences in CD38 remained significant when groups were defined as sex workers vs. controls (see Supplementary Table S2). We interpret that repeated exposure to semen may induce chronic conditioning effects that differs from the acute effects of semen in induction of a potent inflammatory-like response $\mathrm{e}^{13,14}$ in the female reproductive tract. It remains to be determined whether the reduction of immune activation-associated gene expression observed in FSWs is the result of a counter-regulatory response to repeated semeninduced inflammatory changes or owing to IL-10, prostaglandin $\mathrm{E} 2$, and transforming growth factor- $\beta$ in semen. ${ }^{15-18}$ Of interest, we detected higher cervico-vaginal fluid IL-1 $\beta$, IL-6, and IL-8 in women reporting recent condomless sexual activity (see Supplementary Figure 3), supporting our interpretation that differential gene expression induced by chronic semen exposure does not exclude short-term semen driven effects. Although the biological mechanisms underlying the observed lower activation in FSWs remain unknown, exposure to semen induced IFNE in vitro supporting the correlation between recent semen exposure and IFNe expression in vivo.
The detection of IFN $\varepsilon$ among FSWs in our study population establishes for the first time that this type I IFN is expressed in human mucosal tissues. Despite the apparent elevated expression of IFN $\varepsilon$ in epithelium layer, overall tissue RNA analysis did not show an increase in IFN-regulated genes, supporting that its activity may be restricted to cells proximal to the basal membrane as opposed to affecting gene expression across the entire stroma (i.e., largest area within biopsy). Although addressing the direct antiviral effect of semen-induced IFN $\varepsilon$ expression within the cervico-vaginal mucosa is beyond the scope of this report, we hypothesize that IFN $\varepsilon$ might target cells crossing the basal membrane into the epithelium, such as T-cells, DCs, and macrophages. Apart from IFNe expression or lower immune activation, our data also indicate that expression of genes required for HIV infection ${ }^{36}$ (including CD4 (receptor for HIV-1), C-X-C chemokine receptor 4 (co-receptor for HIV), and nucleoporin 153 (required for HIV-1 nuclear import and integration) ${ }^{37}$ ) are downregulated in FSWs, introducing another potential barrier to a productive infection. Lower immune activation and lower expression of genes required for HIV-1 infection together with the increased expression of epithelial IFN $\varepsilon$, suggests that the cervico-vaginal tissue of FSWs may provide for a higher inherent level of resistance to HIV-1 infection. Although direct comparisons of our cervical biopsy gene array data to gene expression from isolated cervical mononuclear cells obtained by cytobrush of the ectocervix (Majengo FSW cohort) is limited by different tissue site collection, ${ }^{9,10}$ similar inferences are possible between studies. Our data and that from the Majengo FSWs both indicate a lower immune activation state in leukocytes from FSW cohorts. ${ }^{10}$ Future studies from tissue-derived $\mathrm{CD} 4{ }^{+}$and $\mathrm{CD} 68^{+}$ cells will be needed to establish direct evidence of HIV resistance within target cells.

Although at a population level condomless sex work does increase HIV seroconversion risk, ${ }^{39,40}$ it remains to be determined whether factors identified here limit the potential frequency of HIV infection, as suggested by earlier unconfirmed reports where previously characterized HESNs became infected following a lapse in sex work. ${ }^{6}$ Our work raises the hypothesis that a lapse in continued maintenance of factors described here (i.e., stop semen exposure) may contribute to a change in susceptibility for HIV infection. ${ }^{6}$ As the role of semen as agonist or antagonist to SIV/HIV-1 transmission remains controversial, ${ }^{21,41}$ studies based on non-human primate models and/or longitudinal human HESN FSW cohorts will be needed to directly address the role of semen and factors described here in mediating and sustaining resistance against SIV-1/HIV-1 infection.

Unlike prior studies in HESN FSW cohorts, our data does not support that sex work alone accounts for previous observations of a higher frequency of FoxP3 $+\mathrm{CD} 4{ }^{+}$T-regulatory cells (Table 2) or differential cervico-vaginal cytokine levels between the control and FSW groups. ${ }^{7,10}$ In addition, prior work in the Majengo FSW HESN cohort found higher CD4 ${ }^{+}$T-cells within cervical scrapings ${ }^{9,42}$ and lower $\mathrm{CD} 4^{+}$T-cell infiltration as indicated by staining of ectocervical biopsies. ${ }^{24}$ The latter results contrast with our findings of increased $\mathrm{CD} 4^{+} \mathrm{T}$-cells, 
macrophages, and plasmacytoid DCs in ectocervical tissue biopsies. Taken together, this suggests that other cohortspecific factors (e.g., HIV exposure, sexual practices, ${ }^{5,43}$ regional, cultural, or genetic factors, etc.) may account for the differences between our and prior studies. For example, differences in reported douching frequency in Africa vs. Latin American women ${ }^{5,43}$ remains to be evaluated as a potential reason for noted differences. It is also important to note that inclusion criteria excluded women showing active local inflammation. Therefore, our findings are limited to constitutive factors that may be associated with a high degree of semen exposure or active sex work absent an ongoing symptomatic infection. Another important limitation of this and other similar studies is that self-reported behavior is frequently inaccurate as has been shown by assessing condom use in HIV-1 prevention/acquisition in multiple studies; ${ }^{44}$ however, studies have also shown that self-reporting of recent coital activity tends to be more accurate. ${ }^{45}$ We interpret that self-reporting in our cohort is more accurate per recent coital exposure as reflected by the observation that recent condomless sexual activity correlated with IFN $\varepsilon$ expression (Figure 3f). We do not address the potential role of semen exposure from multiple donors in FSWs as compared with controls who have a single partner (or semen source). In order to assess the importance of sexual frequency or partner-specific differences in semen composition as potential determinants for the immunological and mucosal differences ascribed to FSWs in this work, future studies will need to define what components of semen or sex work contribute to these changes.

In conclusion, our data bring together for the first time the detection of three separate anti-HIV features, independent of antiviral adaptive immune responses associated with long-term sex work and independent of anti-HIV adaptive responses associated with high-risk HIV exposure. Behavior (long-term sex work), chronic semen exposure, and induction of IFNE within human ectocervical tissue are identified as determinants of local mucosal tissue and systemic changes consistent with decreased HIV-1 susceptibility, which may in part explain sustained HIV-1 HESN status identified in some HIV-1 highrisk cohorts. We propose a working model (see Supplementary Figure S5) suggesting that prolonged sex work can sustain changes in the cervico-vaginal microenvironment that may increase HIV-1 resistance, in part dependent on continued semen exposure. Importantly, the indication that sex work is known to be associated with a higher risk for HIV infection owing to multiple inter-related factors is not addressed here (co-infections, drug use, etc.), ${ }^{2,46}$ therefore limiting our data to documenting host factors that may reduce but not eliminate susceptibility of HIV infection in FSWs.

\section{METHODS}

Participants and ethics statement. All female recruitment carried out for this study was according to Institutional Review Boardapproved protocols at the Wistar Institute (no. 2908242) and the University of Puerto Rico (no. 1350210). All participants gave written informed consent before study inclusion.
Cohort. A total of 85 women were recruited. Fifty FSWs were recruited by the Clinical Research Unit of the Maternal Infant Studies Center (CEMI) at the University of Puerto Rico in San Juan. Thirty-five CG women were recruited from the family planning clinic at CEMI.

Inclusion/exclusion criteria. All recruited women were $\geqslant 21$ years $(21$ years is the legal lower limit for Puerto Rico); gave informed consent; not currently menstruating; tested negative for HIV-1 by OraQuick (Orasure Tech, Bethlehem, PA) and/or PCR; refrained from injection drug use; and completed sexual behavior questionnaire and STI screening.

Recruitment. Recruitment and enrollment of FSWs and women for the control/CG was carried out at the Puerto Rico study site that was simultaneously recruiting FSWs for the HIV Vaccine Trials Network's 907 protocol. $^{25}$ Similar recruiting strategies were employed with the exception that high-risk women actively using injection drugs were excluded. Recruited FSWs were HIV-1 seronegative women who had maintained self-reported high levels of sexual activity (condomless sex with multiple partners) for at least 2 years prior to study inclusion (median of 8 years in sex work as noted in Table 1). Women in the control or CG were enrolled from women attending the women's health clinic at CEMI and reported being in monogamous relationships.

Study visit/sample collection. All consenting women underwent two study visits. An initial visit to collect sex behavior history information, confirm HIV-1 IgG seronegativity, collect peripheral blood for STIs tests (gonorrhea, syphilis, and chlamydia); complete a vaginal $\mathrm{pH}$, and a pap smear test to assess the presence of cervical dysplasia and inflammation. At the second visit, women were counseled on test results and those confirmed to be seronegative for HIV-1 without the presence of active STIs and without a CIN2 or greater dysplastic cytology diagnosis were recruited to the assigned study groups and underwent a sampling for peripheral blood (PBMC archiving and whole-blood phenotypic flow cytometric staining) and cervical fluid collection by vaginal wash with $1 \times$ phosphate-buffered saline (HIV-1specific IgA quantification and cytokine testing). In a subset of FSW and CG women, additional consent was obtained for a colposcopy to collect a cervical biopsy $\left(3 \times 3 \mathrm{~mm}^{2}\right)$ targeting the squamo-columnar junction region otherwise routinely taken as part of standard gynecological care. Biopsies were fixed using Safe Fix II (Fisher Scientific, Pittsburgh, PA) according to the manufacturer's protocol and paraffin embedded. Women were advised to refrain from sexual activity for 1 week until healed; although previous work has shown that such biopsies can be taken safely from women at risk of HIV-1 infection. ${ }^{47}$

Hormone measurements. Archived sera were used to determine the estradiol concentration of participants at the time of blood draw. Hormone concentrations were measured by enzyme-amplified chemiluminescence (Immulite 1,000; Siemens, Munich, Germany) as described by the manufacturer's protocol. The analytical limits of sensitivity of the estradiol and progesterone assays were $15 \mathrm{pg} \mathrm{ml}^{-1}$ (reference range of $20-2,000 \mathrm{pg} \mathrm{ml}^{-1}$ ) and $0.1 \mathrm{ng} \mathrm{ml}^{-1}$ (reference range of $0.2-40 \mathrm{ng} \mathrm{ml}^{-1}$ ), respectively.

Whole-blood staining of lymphocyte subsets. In all, $200 \mu \mathrm{l}$ of whole blood from FSW and CG women was stained for natural killer cell, T-cell, and DC subsets using antibodies obtained from BD Bioscience (San Jose, CA) or Miltenyi (Auburn, CA). Following staining, red blood cells were lysed according to protocol for BD FACS Lysing Solution. Cells were suspended in BD Staining Buffer (BD, Bioscience) for flow cytometric analysis.

PBMC isolation. Standard density gradient centrifugation was used to isolate PBMC from whole blood derived from FSW and CG participants. Briefly, blood was overlaid on Ficoll-pacque Plus (GE Healthcare, Little Chalfont, UK) and centrifuged at $1,200 \mathrm{~g}$ without break. Resulting PBMC were removed and washed in $1 \times$ phosphatebuffered saline. Cells were diluted in trypan blue to exclude dead cells and contaminating red blood cells and then counted. 
HIV-specific T-cell memory. A total of 500000 PBMCs from FSW and CG participants were incubated for $17 \mathrm{~h}$ at $37^{\circ} \mathrm{C}$ with 15 -mer HIV-1 consensus B gag peptides or immunodominant peptides (9-mer) from cytomegalovirus, Epstein-Barr virus, and Influenza obtained through the AIDS Reagent Program, Division of AIDS, NIAID, NIH. For costimulation, CD28/CD49 (BD Bioscience) was added along with Brefeldin A for intracellular staining. Following incubation, cells were stained for CD3 (SK7), CD4 (SK3), CD8 (RPA-T8), and CD107 (H4A3). Following extracellular staining, cells were fixed and permeabilized according to the manufacturer's protocol for BD Cytofix/ Cytoperm (BD Bioscience). Cells were then intracellularly stained for IFN $\gamma$ (25723.11). Finally, cells were suspended in BD Staining Buffer (BD Bioscience) with at least 200000 single cell events collected per target gating. Analysis was carried out with the FlowJo software (Tree Star, Ashland, OR). A positive HIV-1 gag response was defined as $3 \times$ the median or greater of IFNg $+\mathrm{CD}^{+}$T-cells from the CG.

Binding antibody multiplex assay. Mucosal HIV-1 envelopespecific antibodies from FSW and CG CVL samples were measured by a custom HIV-1-binding antibody multiplex assay against a panel of group $\mathrm{M}$ consensus and clade $\mathrm{B}$ envelope glycoproteins as previously described ${ }^{48}$ and are included in Supplementary Methods. Samples were considered positive for HIV-1-specific antibody if they were positive for specific activity for $>2 \mathrm{HIV}-1$-specific antigens.

Cytokine multiplex assay. Life Tech Human Cytokine 30-plex Assay (Carlsbad, CA) was used to measure cervico-vaginal cytokine levels according to the manufacturer's protocol. Data were acquired using a Bio-Plex 200 System (Bio-Rad Laboratories, Hercules, CA). Cutoffs were determined as $50 \%$ of the mean fluorescent intensity of the lowest point on the standard curve for a given cytokine.

Ectocervical biopsy tissue staining. Briefly, $6-\mu \mathrm{m}$ sections were cut and adhered to silanized slides from paraffin-embedded tissues. Immunohistochemical staining for CD123, CD68, CD4, IFNe, and $\mathrm{Mx} 1$ were conducted as previously reported. ${ }^{22,23,32}$ Quantification of tissue staining was carried out as described previously ${ }^{23,32}$ with complete methods provided in Supplementary Methods.

Gene and miRNA expression analyses of ectocervical tissue. Total RNA from formalin-fixed, paraffin-embedded tissues was isolated with High Pure RNA Paraffin Kit (Roche Applied Science, Branford, CT). Total RNA at $500 \mathrm{ng}$ was used on Illumina Whole Genome gene expression DASL HT assay (Illumina, San Diego, CA) with the MCS4 cDNA synthesis reagents and Illumina HT12v4 DASL BeadChip. miRNA expression in Exctocervical Tissues were assayed using TaqMan OpenArray RT PCR platform (Applied Biosystems, Waltham, MA). Information on methods of gene expression data analysis is provided in Supplementary Methods.

Semen preparation. De-identified, discarded cryopreserved semen samples were received under Institutional Review Board exception from the Andrology Laboratory of Penn Fertility Care, University of Pennsylvania. Thirty individual vials of semen were thawed at $37^{\circ} \mathrm{C}$ and pooled into a master mix before refreezing and storage at $-80^{\circ} \mathrm{C}$ until rethawed at $37^{\circ} \mathrm{C}$. Seminal plasma was separated from sperm by centrifugation.

ECT1 culture and immunofluorescence staining for IFN $\varepsilon$. ECT1 cells (ATCC, Manassas, VA) were cultured until 50\% confluent in Keratinocyte-Serum Free Media (Life Technologies, Carlsbad, CA). Cells were then treated with $5 \%$ seminal plasma in Keratinocyte-Serum Free Media (v/v) for $6 \mathrm{~h}$ at $37^{\circ} \mathrm{C}, 5 \% \mathrm{CO}_{2}$. After treatment, cells were fixed in $2 \%$ paraformaldehyde for $20 \mathrm{~min}$. Cells were blocked with $5 \%$ bovine serum albumin $/ 0.2 \%$ Triton $\mathrm{X}$ and incubated with IFN $\varepsilon$ antibody (1:500; Atlas Antibodies, Stockholm, Sweden) overnight. After washing, cells were incubated with an Alexa Fluor 488 Goat Anti-Rabbit IgG $(\mathrm{H}+\mathrm{L})$ Antibody (1:200; Life Technologies). Cells were counterstained with DRAQ5 (1:1,000; Biostatus, Shepshed, UK). Images were collected using a TE2000 Inverted Microscope (Nikon, Melville, NY).
ECT1 RNA Isolation and qRT-PCR. Seminal plasma treated (as above) and untreated ECT1 cells were used for RNA isolation by RNeasy Mini Kit (Qiagen, Valencia, CA). NanoDrop 1000 (ThermoScientific, Waltham, MA) was used to measure RNA quality and quantity. cDNA was prepared from $1 \mu \mathrm{g}$ total RNA using iScript cDNA Synthesis Kit (Bio-Rad) and diluted 1:15 in RNAse-free water. Each PCR sample was tested in triplicate, and the PCR for the housekeeping gene Beta-Actin and the target gene, IFNe, were run in parallel on the same plate. Primers used and information on PCR analysis is provided in Supplementary Methods.

Statistical analysis. Shapiro-Wilk tests were carried out to detect normality distribution of variables and then to determine appropriate statistical tests or procedures. When specified, Fisher's Exact Test was used to analyze contingency tables. Correlations were carried out using the Pearson or Spearman method depending on the previous normality test of variable distribution. $P$-values $<0.05$ were considered significant for single test. For comparisons in each data assay (aside from gene expression analysis described above), when multiple testing adjustment was needed, Benjamini-Hochberg and Benjamini-Yekutieli adjustment were performed at $10 \%$ of the false-discovery rate.

To study the robustness of the results to potential confounding variables, we tested for group differences using a novel, weighted version of the Mann-Whitney test. Individuals were weighted in such a way so that the observed difference between groups is not attributable to the measured confounding variables. The method is based on inverse probability weighting, ${ }^{49,50}$ and a permutation testing approach was used to obtain the $P$-values. Propensity scoring used to derive inverse probability weighting used confounding variables, including marijuana use, vaginal $\mathrm{pH}$, male condom use, and oral contraceptive use.

Significance of overlap between any two gene sets were carried out using hypergeometrical test as a probability of having overlap $\geqslant m$ between sets of size $n_{1}$ and $n_{2}$ with total number of available genes $N$, where $N=$ total number of genes overlapped between two platforms used to generate the two gene lists, $n_{1}=$ number of genes in list 1 , $n_{2}=$ number of genes in list 2 , and $m=$ number of overlapped genes.

SUPPLEMENTARY MATERIAL is linked to the online version of the paper at http://www.nature.com/mi

\section{ACKNOWLEDGMENTS}

We thank R Glenn Overman, Judith Lucas, Audrey Lan, and Matthew Fair for expert technical assistance. This work was funded by NIH grants R01 Al084142 and R01 Al094603 to C.Z. and L.J.M. and T32 Al070099 to S.A.A. Additional support was provided by The Philadelphia Foundation (Robert I Jacobs Fund), Henry S Miller, Jr and J Kenneth Nimblett, AIDS funds from the Commonwealth of Pennsylvania, from the Commonwealth Universal Research Enhancement Program, Pennsylvania Department of Health, the Penn Center for AIDS Research (P30 Al 045008), Wistar Cancer Center Grant (P30 CA10815), and Duke University Center for AIDS Research (CFAR) Immunology Core NIH Grant (5P30 Al064518).

\section{DISCLOSURE}

The authors declared no conflict of interest.

c) 2016 Society for Mucosal Immunology

\section{REFERENCES}

1. Baral, S. et al. Burden of HIV among female sex workers in low-income and middle-income countries: a systematic review and meta-analysis. Lancet Infect. Dis. 12, 538-549 (2012).

2. Sewankambo, N. et al. HIV-1 infection associated with abnormal vaginal flora morphology and bacterial vaginosis. Lancet 350, 546-550 (1997).

3. Naranbhai, V. et al. Innate immune activation enhances hiv acquisition in women, diminishing the effectiveness of tenofovir microbicide gel. J. Infect. Dis. 206, 993-1001 (2012). 
4. Rowland-Jones, S.L. et al. Cytotoxic Tcell responses to multiple conserved HIV epitopes in HIV-resistant prostitutes in Nairobi. J. Clin. Invest. 102, 1758-1765 (1998).

5. Horton, R.E. et al. Cervical HIV-specific IgA in a population of commercial sex workers correlates with repeated exposure but not resistance to HIV. AIDS Res. Hum. Retroviruses 25, 83-92 (2009).

6. Kaul, R. et al. Late seroconversion in HIV-resistant Nairobi prostitutes despite pre-existing HIV-specific CD8 ${ }^{+}$responses. J. Clin. Invest. 107, 341-349 (2001).

7. Card, C.M. et al. Decreased immune activation in resistance to HIV-1 infection is associated with an elevated frequency of CD4(+)CD25(+) FOXP3(+) regulatory T cells. J. Infect. Dis. 199, 1318-1322 (2009).

8. Begaud, E. et al. Reduced CD4 T cell activation and in vitro susceptibility to HIV-1 infection in exposed uninfected Central Africans. Retrovirology 3, 35 (2006).

9. Lajoie, J. et al. Association of sex work with reduced activation of the mucosal immune system. J. Infect. Dis. 210, 319-329 (2014).

10. Chege, D. et al. Blunted IL17/IL22 and pro-inflammatory cytokine responses in the genital tract and blood of HIV-exposed, seronegative female sex workers in Kenya. PLoS One 7, e43670 (2012).

11. Ball, T.B. et al. Polymorphisms in IRF-1 associated with resistance to HIV-1 infection in highly exposed uninfected Kenyan sex workers. AIDS 21, 1091-1101 (2007).

12. Iqbal, S.M. et al. Elevated elafin/trappin-2 in the female genital tract is associated with protection against HIV acquisition. AIDS 23, 1669-1677 (2009).

13. Sharkey, D.J., Tremellen, K.P., Jasper, M.J., Gemzell-Danielsson, K. \& Robertson, S.A. Seminal fluid induces leukocyte recruitment and cytokine and chemokine mRNA expression in the human cervix after coitus. J. Immunol. 188, 2445-2454 (2012).

14. Sharkey, D.J., Macpherson, A.M., Tremellen, K.P. \& Robertson, S.A. Seminal plasma differentially regulates inflammatory cytokine gene expression in human cervical and vaginal epithelial cells. Mol. Hum. Reprod. 13, 491-501 (2007).

15. Robertson, S.A. et al. Seminal fluid drives expansion of the CD4 ${ }^{+} \mathrm{CD} 25+$ $T$ regulatory cell pool and induces tolerance to paternal alloantigens in mice. Biol. Reprod. 80, 1036-1045 (2009).

16. Remes Lenicov, F. et al. Semen promotes the differentiation of tolerogenic dendritic cells. J. Immunol. 189, 4777-4786 (2012).

17. Robertson, S.A., Prins, J.R., Sharkey, D.J. \& Moldenhauer, L.M. Seminal fluid and the generation of regulatory Tcells for embryo implantation. Am. J. Reprod. Immunol. 69, 315-330 (2013).

18. Kafka, J.K. et al. Latent TGF-beta1 is compartmentalized between blood and seminal plasma of HIV-positive men and its activation in semen is negatively correlated with viral load and immune activation. Am. J. Reprod. Immunol. 73, 151-161 (2014).

19. Moonis, M., Lee, B., Bailer, R.T., Luo, Q. \& Montaner, L.J. CCR5 and CXCR4 expression correlated with X4 and R5 HIV-1 infection yet not sustained replication in Th1 and Th2 cells. AIDS 15, 1941-1949 (2001).

20. Munch, J. et al. Semen-derived amyloid fibrils drastically enhance HIV infection. Cell 131, 1059-1071 (2007).

21. Munch, J. et al. Effect of semen and seminal amyloid on vaginal transmission of simian immunodeficiency virus. Retrovirology 10, 148 (2013).

22. Li, Q. et al. Glycerol monolaurate prevents mucosal SIV transmission. Nature 458, 1034-1038 (2009).

23. Abdulhaqq, S.A. et al. Serial cervicovaginal exposures with replicationdeficient SIVsm induce higher dendritic cell $(\mathrm{pDC})$ and $\mathrm{CD} 4^{+} \mathrm{T}$-cell infiltrates not associated with prevention but a more severe SIVmac251 infection of rhesus macaques. J. Acquir. Immune Defic. Syndr. 65, 405-413 (2014).

24. Hirbod, T. et al. Stable CD4 expression and local immune activation in the ectocervical mucosa of HIV-infected women. J Immunol 191, 3948-3954 (2013).

25. Deschamps, M.M. et al. Recruitment of Caribbean female commercial sex workers at high risk of HIV infection. Rev. Panam Salud Publica 34, 92-98 (2013).
26. Shannon, K. et al. Global epidemiology of HIV among female sex workers: influence of structural determinants. Lancet 385, 55-71 (2014).

27. Jennes, W. et al. HIV-specific T helper responses and frequency of exposure among HIV-exposed seronegative female sex workers in Abidjan, Cote d'Ivoire. J. Infect. Dis. 189, 602-610 (2004).

28. Camara, M. et al. Low-level CD4 ${ }^{+} \mathrm{T}$ cell activation in HIV-exposed seronegative subjects: influence of gender and condom use. J. Infect. Dis. 201, 835-842 (2010).

29. Koning, F.A. et al. Low-level CD4 ${ }^{+}$T cell activation is associated with low susceptibility to HIV-1 infection. J. Immunol. 175, 6117-6122 (2005).

30. Hertzog, P., Forster, S. \& Samarajiwa, S. Systems biology of interferon responses. J. Interferon Cytokine Res. 31, 5-11 (2011).

31. Fung, K.Y. et al. Interferon-epsilon protects the female reproductive tract from viral and bacterial infection. Science 339, 1088-1092 (2013).

32. Demers, A. et al. The mucosal expression pattern of interferon-epsilon in rhesus macaques. J. Leukoc. Biol. 96, 1101-1107 (2014).

33. Fellay, J. et al. A whole-genome association study of major determinants for host control of HIV-1. Science 317, 944-947 (2007).

34. Zhou, H. et al. Genome-scale RNAi screen for host factors required for HIV replication. Cell Host Microbe. 4, 495-504 (2008).

35. König, R. et al. Global analysis of host-pathogen interactions that regulate early-stage HIV-1 replication. Cell 135, 49-60 (2008).

36. Brass, A.L. et al. Identification of host proteins required for HIV infection through a functional genomic screen. Science 319, 921-926 (2008).

37. Di Nunzio, F. et al. Nup153 and Nup98 bind the HIV-1 core and contribute to the early steps of HIV-1 replication. Virology 440, 8-18 (2013).

38. Ratto, M.H. et al. The nerve of ovulation-inducing factor in semen. Proc. Natl. Acad. Sci. USA 109, 15042-15047 (2012).

39. Pruss-Ustun, A. et al. HIV due to female sex work: regional and global estimates. PLoS One 8, e63476 (2013).

40. Papworth, E. et al. Epidemiology of HIV among female sex workers, their clients, men who have sex with men and people who inject drugs in West and Central Africa. J. Int. AIDS Soc. 16, 18751 (2013).

41. Allen, S.A. et al. Characterization of the influence of semen-derived enhancer of virus infection on the interaction of HIV-1 with female reproductive tract tissues. J. Virol. 89, 5569-5580 (2015).

42. Iqbal, S.M. et al. Elevated T cell counts and RANTES expression in the genital mucosa of HIV-1-resistant Kenyan commercial sex workers. J. Infect. Dis. 192, 728-738 (2005).

43. Cottrell, B.H. An updated review of of evidence to discourage douching. MCN Am. J. Matern. Child Nurs. 35, 102-107 (2010).

44. Evans, J.L., Couture, M.C. \& Stein, E.S. Biomarker validation of recent unprotected sexual intercourse in a prospective study of young women engaged in sex work in Phnom Penh, Cambodia. Sex. Transm. Dis. 40, 462-468 (2013).

45. Penrose, K.J. et al. Y chromosome and HIV DNA detection in vaginal swabs as biomarkers of semen and HIV exposure in women. Sex. Transm. Dis. 41, 674-679 (2014).

46. Laga, M. et al. Non-ulcerative sexually transmitted diseases as risk factors for HIV-1 transmission in women: results from a cohort study. AIDS 7 , 95-102 (1993).

47. Hasselrot, K. et al. Feasibility and safety of cervical biopsy sampling for mucosal immune studies in female sex workers from Nairobi, Kenya. PLOS One 7, e47570 (2012).

48. Tomaras, G.D. et al. Initial B-cell responses to transmitted human immunodeficiency virus type 1: virion-binding immunoglobulin M (IgM) and IgG antibodies followed by plasma anti-gp41 antibodies with ineffective control of initial viremia. J. Virol. 82, 1244912463 (2008).

49. Lunceford, J.K. \& Davidian, M. Stratification and weighting via the propensity score in estimation of causal treatment effects: a comparative study. Stat. Med. 23, 2937-2960 (2004).

50. Austin, P.C. A tutorial and case study in propensity score analysis: an application to estimating the effect of in-hospital smoking cessation counseling on mortality. Multivariate Behav. Res. 46, 119-151 (2011). 